Service social

\title{
Maintien à domicile : capacité d'offre de services communautaires actuels et futurs au Nouveau-Brunswick
}

\section{Danielle Thériault et Suzanne Dupuis-Blanchard}

Volume 63, numéro 2, 2017

URI : https://id.erudit.org/iderudit/1046505ar

DOI : https://doi.org/10.7202/1046505ar

Aller au sommaire du numéro

Éditeur(s)

École de travail social et de criminologie de l’Université Laval

ISSN

1708-1734 (numérique)

Découvrir la revue

Citer cet article

Thériault, D. \& Dupuis-Blanchard, S. (2017). Maintien à domicile : capacité d'offre de services communautaires actuels et futurs au Nouveau-Brunswick. Service social, 63(2), 143-159. https://doi.org/10.7202/1046505ar
Résumé de l'article

La présente étude comporte trois objectifs : 1) déterminer l'état actuel des services offerts pour le maintien à domicile; 2) explorer la nature de la planification des services futurs pour le maintien à domicile; et 3) exposer les défis reliés aux services et les moyens qui s'offrent aux organismes pour que les services répondent le plus efficacement possible aux besoins des personnes âgées francophones. De type séquentiel exploratoire mixte, cette étude a permis de recueillir des données qualitatives pour bonifier des résultats quantitatifs. Quarante-neuf gestionnaires ont répondu au questionnaire en ligne et 11 entrevues ont été réalisées. Huit thèmes communs ont été dégagés en lien avec les objectifs, thèmes qui montrent une préoccupation pour la viabilité des services communautaires en maintien à domicile. 


\title{
Maintien à domicile : capacité d'offre de services communautaires actuels et futurs au Nouveau-Brunswick
}

\author{
THÉRIAULT, Danielle, BA (psychologie), MGSS \\ DUPUIS-BLANCHARD, Suzanne*, BScInf, MN, PhD
}

RÉSUMÉ

La présente étude comporte trois objectifs : 1) déterminer l'état actuel des services offerts pour le maintien à domicile; 2) explorer la nature de la planification des services futurs pour le maintien à domicile; et 3) exposer les défis reliés aux services et les moyens qui s'offrent aux organismes pour que les services répondent le plus efficacement possible aux besoins des personnes âgées francophones. De type séquentiel exploratoire mixte, cette étude a permis de recueillir des données qualitatives pour bonifier des résultats quantitatifs. Quarante-neuf gestionnaires ont répondu au questionnaire en ligne et 11 entrevues ont été réalisées. Huit thèmes communs ont été dégagés en lien avec les objectifs, thèmes qui montrent une préoccupation pour la viabilité des services communautaires en maintien à domicile.

Mots clés : personnes âgées, maintien à domicile, planification, gestion, organismes communautaires, francophones

\begin{abstract}
The objectives for this study were to: 1) determine the current status of services offered for aging in place; 2) explore the level of planning for future services for aging in place; and 3) depict the challenges related to services and the measures taken by organizations to meet the needs of French speaking older adults. This mixed exploratory sequential study allowed for qualitative data to be collected to better understand the quantitative results. Forty-nine administrators completed the on-line survey and 11 interviews were conducted. Eight common themes emerged in accordance with the study objectives with emphasis on the viability of community services for aging in place.
\end{abstract}

Keywords: older adult, aging in place, planning, administration, community organizations, Francophones 


\section{INTRODUCTION}

La majorité des personnes âgées souhaite demeurer en communauté le plus longtemps possible. En fait, $92 \%$ des personnes âgées de 65 ans ou plus demeurent en communauté au Canada (Statistique Canada, 2012). Cependant, le silence de la Loi canadienne sur la santé au sujet des soins et des services de santé à domicile se traduit par un large écart quant à leur accès (Association canadienne des soins de santé, 2009). La tendance actuelle d'investir des ressources dans les établissements de soins de longue durée a pour effet de créer un déficit en services pour la plupart des personnes âgées qui veulent recevoir des services à domicile. Le modèle des soins institutionnels des années 1970 ne répond plus aux besoins actuels des Canadiens et, surtout, des personnes âgées hors institution.

La province du Nouveau-Brunswick est celle qui compte la plus grande proportion de personnes âgées de plus de 65 ans au Canada, soit 19,9\% (Statistique Canada, 2017). Et parmi ces personnes, 34,5\% sont francophones, ce qui représente le taux le plus élevé de personnes âgées francophones à l'extérieur du Québec (Fédération des aînées et aînés francophones du Canada - FAAFC, 2014). D'autre part, il faut noter que la population francophone est, à l'échelle canadienne, beaucoup plus vieillissante que l'ensemble de la population en général. L'âge médian pour l'ensemble du pays est de 43,7 ans, tandis qu'il est de 48,4 ans pour les francophones (FAAFC, 2014). Les jeunes quittent les régions rurales pour les études postsecondaires et l'emploi, entraînant un véritable exode rural, surtout dans la partie nord de la province où le taux de vieillissement passe à $32 \%$ dans certaines communautés (Statistique Canada, 2017).

Les effets du vieillissement de la population sur les services communautaires se font déjà ressentir. Au Canada, 2,2 millions de personnes bénéficient de soins et de services à domicile (Canadian Medical Association, 2016; Turcotte, 2014) et, entre 2008 et 2011, cette clientèle a connu une augmentation de 55 \% (Association canadienne de soins et services à domicile, 2015). Au Nouveau-Brunswick, une personne âgée sur quatre reçoit des soins à domicile, alors que c'est une personne âgée sur six au Canada (Association canadienne de soins et services à domicile, 2015). Or, au Nouveau-Brunswick, les services pour le maintien à domicile sont majoritairement délivrés par des organismes communautaires non gouvernementaux ayant une entente de services avec le gouvernement provincial, tandis que les soins à domicile sont procurés par les régies de la santé dans le cadre du Programme extra-mural du Nouveau-Brunswick (PEM). La récente stratégie provinciale sur le vieillissement Se tenir ensemble (Gouvernement du Nouveau-Brunswick, 2017) ainsi que l'initiative D'abord chez soi (Gouvernement du Nouveau-Brunswick, 2015) insiste d'ailleurs sur l'importance des services communautaires pour le maintien à domicile. Cependant, le fait que les services communautaires pour le maintien à domicile ne proviennent pas de l'État pose des défis importants quant à l'accès dans la langue officielle de son choix ainsi qu'aux conditions de travail des employés (Bowen, 2015).

La littérature consacrée aux organismes communautaires œuvrant dans le maintien à domicile semble se limiter aux questions suivantes : les modèles de prestation des services (Chen, 2016; Chen et al., 2015; Singelenberg, Stolarz et McCall, 2014; Scharlach, Graham et Lehning, 2012), le soutien communautaire nécessaire (Lum et al., 2016; Szanton et al., 2011), l'application de la technologie et ses avantages (Satariano, Scharlach et Lindeman, 2014) et les services reliés à l'alimentation (Lee, Shannon et Brown, 2015; Lloyd et Wellman, 2015; Wright et al., 2015). Peu de recherches ont été réalisées sur l'état actuel des services et la planification des services futurs de maintien à domicile au Canada, et encore moins au Nouveau-Brunswick. 
Par contre, des études montrent que la majorité des personnes âgées en perte d'autonomie qui demeure chez elles réclament des services à domicile plutôt que des soins infirmiers ou médicaux. L'entretien ménager, le transport et l'aide pour les repas figurent parmi les besoins les plus pressants liés au maintien à domicile des personnes âgées et la prise en charge de la santé (Cao et al., 2014; Dupuis-Blanchard et al., 2013). De plus, certains autres facteurs peuvent déterminer la capacité des personnes âgées à demeurer dans la communauté : la disponibilité de ressources financières, la connaissance et l'utilisation des ressources existantes dans la communauté, la qualité du réseau social et familial ainsi que le réseau des ressources personnelles (Cassity-Caywood et Huber, 2004). Une autre étude (Dupuis-Blanchard et al., 2015) énumère huit stratégies qui s'avèrent favorables au maintien à domicile : la manifestation d'une bonne attitude et d'une détermination bien arrêtée, une perception positive de sa santé, la disponibilité de logements adéquats, l'accès au transport, l'existence d'un réseau de soutien, une communauté accueillante pour les aînés, l'accès à des services, un revenu suffisant et un certain niveau de scolarité.

Vu l'importance des services communautaires pour le maintien à domicile des personnes âgées, la présente étude avait trois objectifs : 1) déterminer l'état actuel des services offerts pour le maintien à domicile; 2) explorer la nature de la planification des services futurs en maintien à domicile; et 3) exposer les défis reliés aux services et les moyens qui s'offrent aux organismes pour que les services répondent le plus efficacement possible aux besoins des personnes âgées francophones, en milieu rural et urbain, en matière de maintien à domicile.

\section{MÉTHODE}

Un devis séquentiel exploratoire mixte (quantitatif-qualitatif) permit de recueillir des données qualitatives pour expliquer et interpréter les résultats quantitatifs (Creswell et Plano Clark, 2010). Deux outils ont été créés, à savoir un questionnaire quantitatif et une grille d'entrevue qualitative. Les outils ont été conçus pour répondre aux objectifs de l'étude tout en s'inspirant à la fois de la littérature pertinente et de l'expérience de l'équipe de recherche.

Le questionnaire quantitatif était composé de 26 questions, en incluant les informations sociodémographiques se rapportant aux répondants. Les questions étaient inspirées par le travail antérieur de l'équipe sur les besoins des personnes âgées pour leur maintien à domicile et portaient sur les types de services actuellement offerts et sur ceux qui seront offerts dans l'avenir pour répondre aux besoins d'ordre physique, psychologique, social, culturel et spirituel, ainsi que sur les services offerts par l'organisme et sur les ressources humaines.

Les participants à l'étude devaient être des gestionnaires responsables d'un organisme communautaire de services de maintien à domicile pour les personnes âgées au Nouveau-Brunswick. Une fois obtenue l'approbation éthique, le recrutement d'un échantillonnage de convenance s'est opéré avec la collaboration d'une association-ressource et à l'aide d'une liste de participants potentiels dressée au moyen d'une recherche en ligne. Deux courriels ont été envoyés, à quelque temps d'intervalle, par l'association-ressource à tous ses membres, les invitant à participer au projet de recherche. Un lien vers le sondage en ligne était fourni dans l'invitation et les personnes intéressées à participer à une entrevue individuelle semi-structurée devaient confirmer leur participation par courriel ou par téléphone à l'une des chercheures. Le schéma d'entrevue était composé de 12 questions complémentaires au sondage quantitatif dans le but de mieux comprendre la planification actuelle et future des services pour le maintien à domicile. 
L'intégralité des données recueillies en ligne (FluidSurveys) ont été entrées dans la version 21 du logiciel SPSS et une analyse de fréquence et de pourcentage a été effectuée - les données manquantes étant exclues de l'analyse. Les entrevues qualitatives réalisées sous forme de rencontres individuelles ont été enregistrées avec le consentement des participants. Elles ont été transcrites puis analysées à l'aide du logiciel NVivo 10 pour en faire ressortir les thèmes les plus communément partagés (Morse et Field, 1995). La saturation des données a été obtenue pour le volet qualitatif.

\section{RÉSULTATS}

En tout, 49 gestionnaires provenant de différents organismes ont participé au questionnaire en ligne et des entrevues ont été réalisées auprès de 11 d'entre eux. Les participants à l'étude étaient majoritairement des femmes âgées de 40 à 59 ans. L'ensemble des données relatives aux gestionnaires qui ont participé au questionnaire en ligne et aux entrevues individuelles $(n=11)$ est présenté au tableau 1. 
Tableau 1. Profil démographique des participants à l'étude

\begin{tabular}{|c|c|c|}
\hline Variables (N) & $\begin{array}{l}\text { Sondage quantitatif } \\
\mathrm{n}(\%)\end{array}$ & $\begin{array}{l}\text { Entrevue } \\
\text { n (\%) }\end{array}$ \\
\hline \multicolumn{3}{|c|}{ Catégories d'âge (47) } \\
\hline Moins de 30 ans & $2(4,3)$ & $0(0,0)$ \\
\hline $31-39$ ans & $4(8,5)$ & $0(0,0)$ \\
\hline $40-49$ ans & $9(19,1)$ & $4(36,4)$ \\
\hline $50-59$ ans & $17(36,2)$ & $4(36,4)$ \\
\hline $60-64$ ans & $6(12,8)$ & $1(9,1)$ \\
\hline 65 ans et plus & $9(19,1)$ & $2(18,2)$ \\
\hline \multicolumn{3}{|l|}{ Sexe (47) } \\
\hline Femme & $36(76,6)$ & $9(81,8)$ \\
\hline Homme & $11(23,4)$ & $2(18,2)$ \\
\hline \multicolumn{3}{|c|}{ Langue(s) parlée(s) (47) } \\
\hline Anglais & $25(53,2)$ & $5(45,5)$ \\
\hline Français & $3(6,4)$ & $0(0,0)$ \\
\hline Anglais et français & $19(40,4)$ & $6(54,5)$ \\
\hline \multicolumn{3}{|c|}{ Niveau de scolarité terminé (47) } \\
\hline Élémentaire & $0(0,0)$ & $0(0,0)$ \\
\hline Secondaire & $3(6,4)$ & $2(18,2)$ \\
\hline Collégial & $16(34,0)$ & $3(27,3)$ \\
\hline Universitaire & $28(59,6)$ & $6(54,5)$ \\
\hline \multicolumn{3}{|c|}{$\begin{array}{l}\text { Nombre d'années comme gestionnaire au } \\
\text { sein de l'organisation (45) }\end{array}$} \\
\hline Moins d'un an & $1(2,2)$ & $1(9,1)$ \\
\hline $1-4$ ans & $9(20,0)$ & $2(18,2)$ \\
\hline $5-9$ ans & $16(35,6)$ & $3(27,3)$ \\
\hline $10-14$ ans & $6(13,3)$ & $2(18,2)$ \\
\hline 15 ans et plus & $13(28,9)$ & $3(27,3)$ \\
\hline \multicolumn{3}{|c|}{$\begin{array}{l}\text { Nombre d'années de travail auprès des aînés } \\
\text { (46) }\end{array}$} \\
\hline Moins d'un an & $0(0,0)$ & $1(9,1)$ \\
\hline $1-4$ ans & $4(8,7)$ & $1(9,1)$ \\
\hline $5-9$ ans & $6(13,0)$ & $0(0,0)$ \\
\hline $10-14$ ans & $5(10,9)$ & $2(18,2)$ \\
\hline 15 ans et plus & $31(67,4)$ & $3(27,3)$ \\
\hline \multicolumn{3}{|c|}{ Type d'organisme (47) } \\
\hline À but non lucratif & $24(51,1)$ & $0(0,0)$ \\
\hline À but lucratif & $23(48,9)$ & $0(0,0)$ \\
\hline \multicolumn{3}{|l|}{ Type de palier (46) } \\
\hline Fédéral & $4(8,7)$ & $0(0,0)$ \\
\hline Provincial & $21(45,7)$ & $5(45,5)$ \\
\hline Régional & $21(45,7)$ & $6(54,5)$ \\
\hline \multicolumn{3}{|c|}{ Clientèle de l'organisme (47) } \\
\hline Urbaine & $9(20,0)$ & $2(18,2)$ \\
\hline Rurale & $8(17,0)$ & $6(54,5)$ \\
\hline Urbaine et rurale & $31(70,0)$ & $3(27,3)$ \\
\hline
\end{tabular}


Les participants aux entrevues individuelles étaient majoritairement des femmes âgées de 40 à 59 ans détenant une formation universitaire. La plupart avaient moins de 5 ans d'expérience en gestion, mais plusieurs années d'expérience dans un travail lié au domaine du vieillissement. Cinq s'identifiaient comme unilingues anglophones et six comme bilingues.

Les résultats des données quantitatives et qualitatives se complètent et, ensemble, ils fournissent une bonne compréhension des réalités et des défis vécus par des gestionnaires d'organismes communautaires offrant des services de maintien à domicile. Les analyses quantitative et qualitative ont permis d'identifier huit thèmes communs.

\section{État actuel des services}

Perceptions à l'égard du système d'offre de services pour le MAD. En général, les participants sont d'avis que le fonctionnement du système actuel fait apparaître des lacunes sérieuses. Certains s'inquiètent de l'accessibilité des services fournis tant aux personnes âgées qu'aux membres de la famille, de la capacité de répondre aux divers besoins de la population vieillissante et de la nature même des soins à domicile.

Parce qu'on n'a pas un système où est-ce qu'on est en mesure de répondre instantanément à un besoin. [...] II faut qu'on change notre modèle de livraison de services parce que ce n'est peut-être pas efficace à ce point-ci. (Entrevue 11)

Quant à l'accessibilité des services, les résultats quantitatifs montrent que, selon la majorité des participants, les personnes âgées ont accès à leurs services soit en téléphonant directement à l'organisme (84\%), à la suite d'une évaluation des besoins à laquelle le ministère du Développement social a procédé (61\%) ou par une ordonnance du médecin ( $41 \%)$.

Sachant que la population est vieillissante, les gestionnaires déclarent qu'il faut agir, en appliquant entre autres les mesures qui sont proposées dans l'initiative D'abord chez soi (Gouvernement du NouveauBrunswick, 2015), tout en conservant les structures qui fonctionnent bien.

Je ne sais pas qu'est-ce que c'était, un remue-méninge ou quelque chose. Je suis arrivée avec ça au début 2000. Dix, quinze ans passés. Comme, comment longtemps que ça prend? Si on attend longtemps assez, ils seront tous morts...tu sais, on attend. II n'y a pas d'action, et c'est ça la partie difficile; et c'est la partie qu'après un bout, pourquoi dire quelque chose, pourquoi tenter de faire quelque chose, pourquoi s'impliquer? Parce qu'il n'y a rien de fait. Je perds mon temps. (Entrevue 6)

État actuel des ressources. Au dire des gestionnaires, la gestion des ressources humaines (la coordination des horaires, l'absentéisme, les conflits interpersonnels, la rétention et le recrutement du personnel) tout comme la pénurie des ressources constituent des défis réels qu'il y a lieu d'affronter pour assurer l'efficacité de l'organisme.

Mais ayant des morceaux d'heures de même, c'est extrêmement dur de coordonner l'horaire d'un employé pour qu'il puisse gagner sa vie. Parce que je fais 2 heures le matin. Tout le monde voudrait de $11 \mathrm{~h}$ à $13 \mathrm{~h}$ par exemple. Bien ça ne se fait pas parce qu'ils ne peuvent pas être partout tout en même temps. (Entrevue 3)

S'il pouvait compter sur plus de ressources, leur organisme, disent-ils, serait en mesure d'offrir à la communauté un plus grand nombre de services. "Je voudrais offrir un service de type autobus à la 
communauté. Mais financièrement, avec tout le restant que l'on fait, on ne peut pas soutenir cela » (Entrevue 5). Pourtant, ils soulignent que la maximisation et l'organisation des ressources de l'organisme permettraient d'accroître son efficacité tout en répondant mieux aux besoins particuliers de la population âgée.

Je crois qu'il y a beaucoup de ressources dans la communauté et s'ils peuvent être créatifs et penser à quelque chose de différent avec les ressources courantes, je crois qu'on peut tous travailler ensemble pour offrir ce qui est nécessaire. Je crois qu'il existe plusieurs occasions pour nous, on doit tout simplement être plus créatif. (Entrevue 1)

\section{Nature de la planification des services}

État des services tant actuels que futurs et qualité des services. Les services à la communauté actuels sont variés. En plus des services de maintien à domicile, certains organismes fournissent des services d'éducation, tant auprès du client que de la famille, de prévention, de transport, d'accompagnement aux rendez-vous, de livraison de repas à domicile, de soutien social et d'offre de matériaux. Certains participants prévoient même des activités sociales pour les personnes âgées : « On a toutes sortes d'activités gratuites ou à frais très minimes à la communauté. Ça peut être des regroupements d'aînés, un club d'âge d'or qui nous demande pour un atelier sur le deuil, sur la dépression, etc. » (Entrevue 7) Quoique les organismes offrent déjà toute une variété de services, des gestionnaires mentionnent aussi certains autres services qu'ils aimeraient pouvoir offrir à la communauté.

Une des choses à laquelle je songe c'est un autobus, utiliser notre autobus pour déplacer les gens à leur rendez-vous médical, la pharmacie, l'épicerie, différents endroits qu'ils iraient normalement, mais ne peuvent plus fréquenter, car ils n'ont aucun transport pour s'y rendre. (Entrevue 1)

Les résultats quantitatifs permettent de préciser les services qui sont actuellement offerts dans la province et la planification des services futurs liés au maintien à domicile.

En ce qui concerne la catégorie de la santé physique (tableau 2), parmi les services qui sont les plus offerts, mentionnons l'offre de conseils et d'information touchant à la santé, les soins concernant des problèmes de santé particuliers, l'aide pour remplir des formulaires, les soins palliatifs ou de fin de vie, l'aide pour la préparation de repas, l'aide pour le ménage de même que l'accompagnement de nuit. Les participants prévoient en outre offrir plus tard d'autres services. La plupart des organismes offrent également aux personnes âgées diverses formes de soutien psychologique. Les résultats sont semblables pour l'offre de services futurs (tableau 3). 
Tableau 2. Fréquence et pourcentage des services relevant de la catégorie physique

\begin{tabular}{|c|c|c|}
\hline Variables & $\begin{array}{l}\text { Actuellement } \\
\text { n (\%) }\end{array}$ & $\begin{array}{l}\text { À l'avenir } \\
\text { n (\%) }\end{array}$ \\
\hline Soins des pieds & $23(47,9)$ & $19(50,0)$ \\
\hline Aide pour remplir des formulaires & $30(63,8)$ & $23(60,5)$ \\
\hline Aide pour le ménage & $25(52,1)$ & $20(51,3)$ \\
\hline Aide pour la préparation de repas & $26(53,3)$ & $20(52,6)$ \\
\hline Tonte du gazon & $11(23,9)$ & $8(20,0)$ \\
\hline Déneigement & $10(21,7)$ & $7(17,5)$ \\
\hline Aide pour le déménagement & $12(25,5)$ & $7(17,9)$ \\
\hline Aide pour les activités de débarras & $20(42,6)$ & $14(35,0)$ \\
\hline Aide pour l'épicerie & $20(43,5)$ & $18(43,9)$ \\
\hline Accompagnement de nuit & $24(51,1)$ & $17(44,7)$ \\
\hline Repas livrés à la maison & $8(17,0)$ & $13(31,7)$ \\
\hline Repas offerts en communauté & $12(25,5)$ & $15(35,7)$ \\
\hline $\begin{array}{l}\text { Offre de conseils et d'information en } \\
\text { santé (prévention, promotion de la santé) }\end{array}$ & $39(81,3)$ & $34(87,2)$ \\
\hline $\begin{array}{l}\text { Soins concernant des problèmes de } \\
\text { santé particuliers }\end{array}$ & $33(70,2)$ & $25(65,8)$ \\
\hline Soutien/soins palliatifs ou de fin de vie & $28(58,3)$ & $23(59,0)$ \\
\hline Centre de jour & $9(18,8)$ & $13(32,5)$ \\
\hline $\begin{array}{l}\text { Centre de renseignements concernant les } \\
\text { services de maintien à domicile }\end{array}$ & $22(46,8)$ & $27(71,1)$ \\
\hline
\end{tabular}

Tableau 3. Fréquence et pourcentage des services relevant de la catégorie psychologique et sociale

\begin{tabular}{|c|c|c|}
\hline Variables & $\begin{array}{l}\text { Actuellement } \\
\text { n (\%) }\end{array}$ & $\begin{array}{l}\text { À l'avenir } \\
\text { n (\%) }\end{array}$ \\
\hline \multicolumn{3}{|l|}{ Psychologique } \\
\hline $\begin{array}{l}\text { Réponse aux appels téléphoniques le soir } \\
\text { et/ou la nuit venant d'aînés soudainement } \\
\text { inquiets }\end{array}$ & $16(33,3)$ & $18(45,0)$ \\
\hline $\begin{array}{l}\text { Disponibilité d'une personne à qui les } \\
\text { aînés peuvent parler de leurs inquiétudes }\end{array}$ & $33(68,8)$ & $28(70,0)$ \\
\hline Accompagnement à des rendez-vous & $24(51,1)$ & $20(51,3)$ \\
\hline $\begin{array}{l}\text { Soutien à la famille de la personne âgée } \\
\text { Sociale }\end{array}$ & $28(58,3)$ & $22(56,4)$ \\
\hline Transport à des rendez-vous médicaux & $24(51,1)$ & $19(48,7)$ \\
\hline $\begin{array}{l}\text { Transport à des activités sociales dans la } \\
\text { communauté }\end{array}$ & $20(41,7)$ & $16(41,0)$ \\
\hline $\begin{array}{l}\text { Activités sociales pour les aînés dans la } \\
\text { communauté }\end{array}$ & $26(54,2)$ & $20(51,3)$ \\
\hline Visites sociales à domicile & $19(39,6)$ & $17(42,5)$ \\
\hline
\end{tabular}


D'après les participants, les services relevant de la catégorie de la santé sociale qui sont actuellement les plus fournis à la population âgée sont les activités sociales et le transport aux rendez-vous médicaux. Ce sont aussi ces services que les participants envisagent d'offrir davantage à l'avenir (tableau 3).

Dans la catégorie du soutien culturel, $85 \%$ des gestionnaires déclarent que leurs services sont offerts en anglais pour les anglophones, tandis que seulement $64 \%$ des gestionnaires indiquent que leurs services sont offerts en français pour les francophones. Peu de changements sont prévus quant à l'offre de services futurs dans la langue maternelle du client ( $84 \%$ et $65 \%$ respectivement).

Environ $36 \%$ des participants offrent le service de prière avec la personne âgée et $30 \%$ l'accompagnement à des services religieux. Les résultats sont semblables pour l'offre de services futurs, soit $31 \%$ et $28 \%$ respectivement.

Même si les gestionnaires offrent une variété de services associés au maintien à domicile, plusieurs se disent insatisfaits des services qu'offre leur organisme. Seulement $58 \%$ des gestionnaires disent être satisfaits de leurs services et $76 \%$ déclarent que les services qu'offre leur organisme permettent aux personnes âgées de bénéficier du maintien à domicile. La nécessité d'offrir plus de services à la communauté fait l'objet de commentaires durant les entrevues.

Je crois qu'ils doivent les remettre à la maison le plus rapidement possible, mais tu ne les envoies pas à la maison sans qu'il y ait du soutien de disponible. Et jusqu'à ce qu'ils commencent à réaliser l'importance de ce soutien, ils ne vont pas le financer et s'ils ne vont pas le financer, ils ne vont pas avoir de proches aidants, et ils vont aboutir avec toute une situation. (Entrevue 9)

Malgré ce constat, $87 \%$ des gestionnaires prévoient réviser l'éventail de leurs services par suite du vieillissement de la population.

Lorsque nous avons posé des questions concernant les langues officielles de la province, nous avons constaté que peu de participants offrent des services bilingues. En fait, $33 \%$ des gestionnaires se disent incapables d'offrir des services dans les deux langues et $55 \%$ d'entre eux disent que leurs employés qui sont capables d'offrir des services en français représentent moins de $30 \%$ du personnel dans leur organisme. Durant les entrevues, certains gestionnaires ont dit que leur organisme compte peu d'employés bilingues ou que, dans certains cas, le client ne demande pas que les services lui soient fournis en français : «Et, quand tu demeures dans cette région, ils parlent anglais $90 \%$ du temps, alors ce n'est pas vraiment un problème. Et, je ne sais pas en termes d'employés, combien sont bilingues » (Entrevue 9). Malgré les difficultés rencontrées, la plupart des gestionnaires sont sensibilisés à l'importance d'une offre de services dans la langue préférée du client : « Nous avons quelques employés qui parlent le français, ce qui est magnifique, alors on peut s'assurer que les personnes francophones connaissent les autres francophones pour qu'ils puissent tous parler la même langue s'ils le désirent » (Entrevue 1).

\section{Les défis et les moyens}

Environnement externe. En plus des défis internes que doit affronter l'organisme, les participants ont aussi signalé ceux que crée l'environnement externe. Selon eux, la demande de soins et de services est à la hausse et elle s'avère de plus en plus complexe : " À l'avenir, il va falloir certainement se pencher là-dessus [complexité des demandes] puis voir comment on peut améliorer ça parce que la 
population augmente, les besoins sont différents »(Entrevue 11). Le contexte démographique de la province vient ajouter des soucis, les réalités étant fort différentes en milieu rural et en milieu urbain.

Mais les défis sont toujours plus dans les régions rurales que dans les régions urbaines, au niveau des services. C'est toujours un défi, parce que c'est beaucoup moins profitable, pour les agences, dans une plus petite région. C'est souvent [des] gens qui vivent dans [des] milieux plus isolés, c'est plus difficile de [les] desservir à domicile. (Entrevue 11)

Les gestionnaires soulignent aussi certaines répercussions d'enjeux concernant l'appareil gouvernemental sur la gestion des organismes, dont les difficultés d'adaptation lorsque survient un changement de gouvernement : « Ça fait trois fois que je pense que j'vais y arriver, puis on change de gouvernement. Ça me prend comme trois ans pour me battre, puis j'arrive à la porte, ils changent de couleur " (Entrevue 7). Dans certains cas, l'admissibilité aux services est restreinte et contribue à l'augmentation des dépenses de la province.

Donc ils sont référés par l'appareil gouvernemental, mais on s'est rendu compte avec le temps que les critères d'admissibilité pour avoir accès [à] la majorité des services sont beaucoup trop serrés. Quand ils arrivent à pouvoir [accéder] aux services, ils sont à la porte du foyer. (Entrevue 7)

Certains participants se plaignent du manque de soutien aux organismes communautaires.

Il y a un roulement très élevé d'employés de soutien à domicile ou d'aidants et un des plus grands facteurs dans cela, si tu veux entendre ceci, est le gouvernement qui abaisse la profession. OK. II ne nous soutient pas comme éducateur; il ne nous soutient pas comme livreur de services en leur nom. (Entrevue 9)

Engagement communautaire et collaboration. Durant les entrevues, l'engagement et la collaboration ont souvent été mentionnés. Les gestionnaires expliquent que c'est grâce à l'engagement communautaire et à la collaboration de la population que certains de leurs services peuvent être offerts aux aînés. Ainsi, plusieurs gestionnaires peuvent offrir des services en recourant au bénévolat communautaire - « la ressource “ bénévole aînés" est une richesse en soi » (Entrevue 7) -, ou encore à des partenariats entre organismes - « Vraiment une bonne collaboration qui est en train de se former et cela va vraiment aider au niveau des personnes âgées pour sûr » (Entrevue 10). Les gestionnaires soulignent l'importance du bénévolat et du travail en commun dans la réponse à donner aux besoins de la population vieillissante.

Certains gestionnaires mentionnent également le soutien intergénérationnel comme moyen de répondre à divers besoins des personnes âgées.

Je regarde, l'été passé, la maison des jeunes a mis en place une coopérative jeunesse qui faisait de la peinture, du coupage de gazon, puis des travaux dans ces genres-là. Puis je me dis, l'organisation, avec sa mise en place, puis la ressource nécessaire, peut s'asseoir avec eux (puis y en a dans différentes régions) : qu'est-ce que ça serait que vous mettiez ça en place à l'année? Pour dire, bon, y a des camelots qui vont passer le journal, y a-tu des jeunes qui seraient intéressés de prendre 3-4 perrons à déneiger? (Entrevue 7)

Inévitablement, les participants déclarent qu'il est important d'inclure les aînés et les autres membres de la communauté dans la prise de décisions concernant la création de programmes et de services, et cela aussi bien pour établir une relation de collaboration que pour approfondir la connaissance de leurs besoins. 
Je voudrais que la communauté soit plus sensibilisée, et plus libre à demander des services. Au lieu de nous qui offre, ils devraient être ceux qui viennent à nous et disent « on aimerait vraiment cela si vous obteniez un autobus ». Bon, si c'est ce que la communauté désire, on peut alors se rallier ensemble pour le même but. (Entrevue 5)

Formation des employés. Un autre point discuté dans les entrevues a trait à la formation des employés. Compte tenu des conditions de travail, de leur salaire et des frais afférents à la formation, les employés manifestent peu d'intérêt pour les activités ou les programmes de formation : "Quand tu as 50 ans et plus et tu fais $12 \$$ de l'heure et tu ne peux pas gagner ta vie, il y en a qui refusent: "Pourquoi passer à travers de ça?" » (Entrevue 3) Les gestionnaires reconnaissent l'importance de la formation du personnel, d'autant plus que les besoins de la population vieillissante se complexifient de plus en plus.

Parce que je crois que les services que nos employés donnent [à domicile] sont certainement, comment je dirais ça, plus en besoin, plus sérieux, plus avancés... Dans le sens que, par le passé, les personnes qui allaient aux fameux « nursing homes », ils étaient là pendant à peu près, je ne dis pas dix ans, mais proche, peut-être sept-huit ans en moyenne, ce qui veut dire qu'ils étaient raisonnablement en bonne santé. À l'heure actuelle c'est moins de deux ans. Ce qui veut dire qu'une fois qu'on les a dans les fameux foyers de soins spéciaux, puis on les a aussi dans les soins à domicile aussi. Le monde est plus malade, naturellement, de là le besoin d'avoir une meilleure formation. (Entrevue 8)

En outre, des gestionnaires insistent sur la valeur ajoutée d'une formation de qualité, qui rendrait ce milieu de travail plus attrayant.

Ça pourrait être le collège qui viendrait en région donner la formation, ou ça pourrait être quelqu'un comme nous autres qui dirait qu'on va mettre ça ensemble, je ne sais pas trop. Mais que par après, ça serait reconnu [et] que le salaire va augmenter à 14 \$ de l'heure. (Entrevue 8)

Conscientisation de la société. Les participants font observer qu'il y a de la préparation à faire auprès de la société. Des changements doivent être apportés relativement à certaines valeurs sociétales et aux mentalités, et les responsabilités individuelles en contexte communautaire doivent être clairement définies : « [...] beaucoup d'emphase sur les hôpitaux, sur les soins tertiaires, et le reste, on n'a rien fait. Mais si qu'on ne change pas notre mentalité, qu'il y ait un virement drastique vers la communauté, on ne va pas faire le croche » (Entrevue 10). Des gestionnaires affirment même que les personnes âgées souvent ne sont pas au courant de l'existence de certains services dont elles pourraient bénéficier.

Tu sais, ils ne savent même pas qu'ils peuvent appeler au programme extra-mural pour la physio ou la diététicienne par eux-mêmes. «Tu veux dire que je peux tout simplement appeler? » Je leur réponds que oui, ils peuvent. «Oh, je ne savais pas ça. » Alors, de petites choses comme ça. Parfois je les dirige vers Sobey's pour parler avec une diététicienne. Ou encore, je leur dis de parler au pharmacien s'ils ont des questions avec leur médicament. Bon, ils n'ont jamais fait cela dans le passé. (Entrevue 6)

Tangibilité du maintien à domicile. Les participants expriment certaines inquiétudes quant à la viabilité du maintien à domicile. Les conditions de travail présentent un enjeu majeur et rendent problématiques la rétention et le recrutement du personnel dans les organismes communautaires.

Mais, je parlais avec un des gestionnaires ici, et il paie le concierge pour laver le plancher quand l'édifice est fermé, et il le paie 25 \$ de l'heure. On paie nos employés 13 \$ de l'heure, pour un travail isolé et 
pour livrer des soins à une personne. Et la formation; il faut avoir de la formation, mais on ne veut pas payer. Alors, si je peux aller au Pizza Delight et faire 50 \$ en pourboire par relais, pourquoi je travaillerais pour $13 \$$ de l'heure? (Entrevue 9)

La grande majorité des participants (69\%) a indiqué que leurs employés reçoivent une rémunération allant de $10,31 \$$ à $15 \$$ de l'heure. Près de $40 \%$ des gestionnaires ne rémunèrent pas les déplacements de leurs employés et $71 \%$ d'entre eux déclarent que leurs employés se servent de leur voiture personnelle pour se rendre chez les clients. De plus, $89 \%$ des gestionnaires mentionnent que la plupart de leurs employés sont des femmes, $51 \%$ que leurs employés ont majoritairement entre 41 et 50 ans et $77 \%$ que leurs employés âgés de moins de 30 ans représentent moins de $30 \%$ du personnel de leur organisme.

Ce n'est pas pour demain, mais peut-être que si on veut que l'industrie connaisse du succès, il faut aller chercher les plus jeunes. Il faut être alerte que le fait que dans ce temps-ci, l'âge moyen est de 50 ans et plus. Où est la relève? II n'y en a pas. Donc il faut vraiment regarder à ça, voir ce qu'on peut faire différemment pour recruter. Autre que ça, on va avoir des problèmes, des gros problèmes, puis on ne les adresse pas. (Entrevue 8)

Malgré tous les défis à relever, les gestionnaires soulignent les dimensions importantes et gratifiantes du maintien à domicile. Les services de maintien à domicile apportent des bienfaits aux aînés et permettent aussi de diminuer les dépenses de la province tout en exerçant un rôle non négligeable sur les services communautaires de la province.

Puis quand tu regardes les centres de jour, les centres de ressources communautaires, la ressource dans la communauté, ça, c'est bâtisseur, c'est guérisseur, c'est préventif. On élimine des problèmes de maladie chronique, on élimine des hospitalisations, on élimine à l'urgence, santé mentale, psychologique. Oui, on a beaucoup de bobos, puis il faut mettre de l'argent dans la sacoche des bobos, mais l'aspect préventif va faire que ça va nous coûter moins cher dans le futur. (Entrevue 7)

\section{Discussion}

Les résultats de l'étude nous permettent d'affirmer que plusieurs difficultés liées aux ressources humaines représentent une menace importante pour l'offre de services de maintien à domicile. Bien que notre étude n'ait pas porté précisément sur l'état des ressources humaines au sein des organismes communautaires, celle-ci a mis en lumière que les conditions de travail des employés, les défis que constituent le recrutement des employés et leur rétention ainsi que la nécessité de mettre en place une formation continue sont des enjeux prioritaires pour les gestionnaires. II y a lieu de s'inquiéter de la viabilité des services communautaires en maintien à domicile puisque l'âge moyen des employés majoritairement des femmes - engagés pour assurer la prestation des services de maintien à domicile est près de la cinquantaine et que très peu d'employés ont moins de 30 ans. De plus, la rémunération est presque celle du salaire minimum $(10,65 \$)$ et la plupart des employés doivent se servir de leur voiture personnelle, souvent sans compensation, pour aller chez leurs clients.

En dépit du fait que les gestionnaires reconnaissent l'importance de la formation des employés, de base ou continue, peu d'employés se montrent intéressés à participer à des activités de formation en raison de leur faible rémunération et de leurs conditions de travail. Des chercheurs (Keefe et al., 2011) constatent le manque d'uniformité dans la formation des employés de soutien de même qu'une disparité des salaires selon les milieux de travail, pour des emplois exigeant par ailleurs le même niveau de 
formation. Cette question des conditions de travail et de la rémunération met très certainement en péril les services communautaires de soutien à domicile des aînés. En fait, la stratégie sur le vieillissement de la province du Nouveau-Brunswick (Gouvernement du Nouveau-Brunswick, 2017) souligne l'importance d'élaborer un vaste plan de ressources humaines et annonce qu'il sera désormais exigé que les programmes de formation de niveau collégial et universitaire incluent des cours dans le domaine du vieillissement dans le but de développer une main-d'œuvre bien formée.

Le fait que les organismes communautaires se trouvent dans l'impossibilité de garantir une offre de services dans la langue du client est préoccupant dans une province officiellement bilingue. Bien que la Loi sur les langues officielles du Nouveau-Brunswick exige que les services hospitaliers et les services gouvernementaux soient fournis dans les deux langues officielles, les organismes communautaires avec entente avec le gouvernement provincial, quant à eux, ne sont nullement tenus d'offrir des services bilingues. Les personnes âgées francophones qui demeurent dans des communautés linguistiques officielles en situation minoritaire au Canada éprouvent depuis longtemps des difficultés d'accès aux services et aux soins en français (Dupuis-Blanchard et al., 2015; 2013). Les gestionnaires expliquent qu'il est difficile de recruter et de retenir les employés bilingues car ces personnes peuvent trouver un emploi ailleurs et profiter de meilleures conditions de travail. Et pourtant, l'offre de services dans la langue du client est essentielle pour assurer des services et des soins sécuritaires, la satisfaction du client et la qualité des services (Réseau des services de santé de l'Est de l'Ontario, 2012).

Un autre constat de l'étude est que, si l'on se fonde sur ce que planifient les gestionnaires, le nombre de services offerts par les organismes communautaires n'augmentera guère dans un proche avenir, ni leur envergure. En fait, seuls quelques services devraient connaître un plus grand développement. En dépit de leur caractère essentiel pour le maintien à domicile des aînés en perte d'autonomie, il n'est pas prévu que les services d'entretien intérieur et extérieur de la maison, de transport et d'aide pour accomplir les tâches quotidiennes soient bonifiés. II se peut que cette situation reflète l'état des ressources humaines ou encore le financement de ces organismes par l'appareil gouvernemental. La littérature sur le sujet nous permet de constater que, depuis plusieurs années maintenant, les tentatives de financement des soins et des services à domicile échouent. Dans le cadre de notre étude, les gestionnaires interviewés ont clairement exprimé leurs frustrations devant cette situation : les progrès réalisés sur le plan des conditions de travail risquent d'être perdus par suite d'un changement de gouvernement ou de ministre. Ils se disent inquiets lorsqu'ils pensent aux services futurs et au vieillissement de la population.

Nos résultats font aussi état de l'importance et de la valeur des liens qui unissent les organismes et la communauté, en particulier sous la forme du bénévolat et de la collaboration ainsi que du partenariat entre organismes. II n'est pas surprenant que les organismes communautaires, qui comptent peu de ressources, valorisent l'appui de la communauté. Le bénévolat a une grande importance dans le soutien aux services de maintien à domicile : le transport, les appels téléphoniques de soutien social et les visites amicales dépendent de personnes non rémunérées. Les adultes de moins de 44 ans sont le groupe d'âge qui contribue le plus au bénévolat, mais ils offrent moins d'heures que les personnes âgées de 65 ans et plus (Vézina et Crompton, 2012). Ces auteurs notent toutefois que les heures de bénévolat au Canada sont à la baisse depuis quelques années - mais non en milieu rural où la contribution des bénévoles et des communautés a augmenté (Vézina et Crompton, 2012; Barr et al., 2004), ce qui, on le présume, vient un peu compenser la plus grande difficulté d'offrir des services de maintien à domicile dans les régions excentrées. 
Le vieillissement de la population et les services communautaires nécessaires pour le maintien à domicile sont des thématiques d'étendue nationale. Notre étude démontre l'importance d'une planification à long terme pour répondre aux besoins d'une population vieillissante. Nous remarquons une ferme volonté de desservir la population âgée de la part des gestionnaires des organismes communautaires. Avec les ressources financières et humaines adéquates, ces organismes pourraient sensibiliser les communautés dans lesquelles ils œuvrent à l'importance de planifier et de préparer le maintien à domicile pour les personnes d'âge avancé, collaborer avec d'autres organismes communautaires pour partager les ressources ainsi que favoriser le retour à domicile à la suite d'une hospitalisation, et jouer un plus grand rôle dans l'engagement des communautés pour soutenir les aînés de leur communauté. Par contre, les résultats de l'étude montrent que les défis sur le plan des ressources humaines perturbent la planification des services actuels et futurs.

\section{Forces et limites de l'étude}

L'approche de recherche mixte a permis de comprendre davantage l'offre de services actuelle et future des organismes communautaires pour le maintien à domicile des aînés au Nouveau-Brunswick. La province du Nouveau-Brunswick est l'endroit idéal pour effectuer de la recherche sur le vieillissement en raison de son profil sociodémographique. Les résultats éclairent les organismes communautaires, les communautés et les décideurs politiques sur la réalité des gestionnaires et pourront appuyer une réflexion sur l'avenir des organismes communautaires en maintien à domicile.

Les limites de l'étude tournent principalement autour du recrutement des participants et de l'échantillonnage de convenance. Le fait qu'il n'existe aucune liste officielle d'organismes communautaires en maintien à domicile peut avoir limité le recrutement de participants potentiels ainsi que l'échantillonnage. Une autre limite est le nombre d'entrevues réalisées, seuls 11 gestionnaires s'étant montrés intéressés à participer à une entrevue semi-structurée avec la première auteure.

\section{Conclusion}

Des changements majeurs doivent être mis en œuvre pour garantir la viabilité des organismes communautaires et la continuité des services de maintien à domicile des personnes âgées. Notre étude ayant pour objet de faire l'état actuel des services et d'explorer la nature de la planification future de ces services, il est important de noter que les ressources humaines représentent la cible principale de toute planification de services de ce genre pour la population vieillissante. Si les conditions de travail ainsi que le recrutement et la rétention des ressources humaines demeurent à l'état actuel, il apparaît inévitable que la viabilité du maintien à domicile soit bientôt mise en question.

Notre étude permet de jeter un nouveau regard sur la planification future des services de maintien à domicile tout en mettant en lumière les défis que doivent affronter les services communautaires. Plusieurs associations nationales et agences gouvernementales appuient le virage de notre système de santé vers la communauté, mais à cette fin, il faudra s'assurer de pouvoir compter sur une maind'œuvre suffisante, bien formée, bilingue, jouissant de conditions de travail acceptables compte tenu de l'importante responsabilité qui lui est confiée dans le maintien de l'autonomie des personnes âgées. 
THÉRIAULT, Danielle, BA (psychologie), MGSS Coordonnatrice à la recherche Centre d'études du vieillissement

DUPUIS-BLANCHARD, Suzanne*, BScInf, MN, PhD Professeure agrégée Titulaire de la Chaire de recherche en santé CNFS-Université de Moncton sur le vieillissement École de science infirmière

Université de Moncton *auteure pour la correspondance

\section{RÉFÉRENCES}

Association canadienne des soins de santé. (2009). Home Care in Canada: From the margins to the mainstream, Ottawa, Association canadienne des soins de santé. [http://www.homecareontario.ca/docs/default-source/publicationsmo/home_care_in_canada_from_the_margins_to_the_mainstream_web.pdf?sfvrsn=6]

Association canadienne de soins et services à domicile. (2015). Home Care in Canada: Advancing quality improvement and integrated care, Ottawa, Association canadienne de soins et services à domicile. [http://www.cdnhomecare.ca/media.php?mid=4328]

Barr, C., McKeown, L., Davidman, K., Mclver, D. et Lasby, D. (2004). A Portrait of the Nonprofit and Voluntary Sector in Rural Ontario, Toronto, Canadian Centre for Philanthropy.

Bowen, S. (2015). Impact des barrières linguistiques sur la sécurité des patients et la qualité des soins, Ottawa, Société Santé en français.

Canadian Medical Association. (2016). The State of Seniors Health Care in Canada, Ottawa : Canadian Medical Association. [https://www.cma.ca/En/Lists/Medias/the-state-of-seniors-healthcare-in-canada-september-2016.pdf]

Cao, M., Guo, X., Yu, H., Chen, L. et McDonald, T. (2014). “ Chinese community-dwelling elders' needs: Promoting ageing in place », International Nursing Review, vol. 61, n 3, p. 327-335.

Cassity-Caywood, W. et Huber, R. (2004). «Rural older adults at home », Journal of Gerontological Social Work, vol. 41, $\mathrm{n}^{\text {os }} 3-4$, p. 229-245.

Chen, S. H. (2016). « Determining the service demands of an aging population by integrating QFD and FMEA method », Quality \& Quantity: International Journal of Methodology, vol. 50, n 1, p. 283298.

Chen, C. K., Shies, A. J., Wang, K. M. et Yu, C. H. (2015). «An ageing-in-place service innovation model using TRIZ methodology », Human Factors and Ergonomics in Manufacturing and Service Industries, vol. 25, n² 2, p. 166-182. 
Creswell, J. et Plano Clark, V. (2010). Designing and Conducting Mixed Methods Research, Thousand Oaks (Californie), Sage Publications.

Dupuis-Blanchard, S., Gould, O., Gibbons, C., Simard, M., Éthier, S., et Villalon, L. (2015). Strategies for Aging in Place: The experience of language-minority seniors with loss of independence. Global Qualitative Nursing Research, Open Access, p. 1-9.

Dupuis-Blanchard, S., Simard, M., Gould, O., et Villalon, L. (2013). La perception des aînés francophones en situation minoritaire face aux défis et aux enjeux liés au maintien à domicile en milieu urbain néo-brunswickois. Revue canadienne de santé publique, vol. 104, nº 6, p. 71-74.

Fédération des aînées et aînés francophones du Canada. (2014). Profil statistique 2011. Personnes âgées francophones au Canada, Ottawa, Fédération des aînées et aînés francophones du Canada. [http://www.faafc.ca/images/memoires/Profil_statistique_-_2011_-.pdf]

Gouvernement du Nouveau-Brunswick. (2017). Se tenir ensemble. Une stratégie sur le vieillissement pour le Nouveau-Brunswick, Fredericton, Gouvernement du Nouveau-Brunswick. [http://www2.gnb.ca/content/dam/gnb/Departments/sd-ds/pdf/Seniors/AnAgingStrategyForNB.pdf]

Gouvernement du Nouveau-Brunswick. (2015). D'abord chez soi, Fredericton, Gouvernement du Nouveau-Brunswick. [http://www2.gnb.ca/content/dam/gnb/Departments/sdds/pdf/DabordChezSoi.pdf]

Keefe, J., Knight, L., Martin-Matthews, A. et Légaré, J. (2011). « Key issues in human resource planning for home support workers in Canada », Work, vol. 40, p. 21-28.

Lee, J., Shannon, J. et Brown, A. (2015). "Characteristics of older Georgians receiving Older Americans Act Nutrition Program Services and other home and community-based services ", Journal of Nutrition in Gerontology and Geriatrics, vol. 34, $n^{\circ}$ 2, p. 168-188.

Lloyd, J. et Wellman, N. (2015). "Older Americans Act Nutrition Programs: A community based nutrition program helping older adults remain at home ", Journal of Nutrition in Gerontology and Geriatrics, vol. 34, n² 2, p. 90-109.

Lum, T., Lou, V., Chen, Y., Wong, G. et Luo, H. (2016). « Neighborhood support and aging-in-place preference among low income elderly Chinese city-dwellers », The Journal of Gerontology Series $B$, vol. 71B, no 1, p. 98-105.

Morse, J. et Field, P. A. (1995). Qualitative Research Methods for Health Professionals, Thousand Oaks (Californie), Sage Publications.

Réseau des services de santé de l'Est de l'Ontario. (2012). Les services en français. Un facteur de qualité des services de santé, Ottawa, Réseau des services de santé de l'Est de l'Ontario. 
Satariano, W., Scharlach, A. et Lindeman, D. (2014). « Aging, place, and technology: Toward improving access and wellness in older populations », Journal of Aging and Health, vol. 26, $\mathrm{n}^{\circ} 8$, p. 1373-1389.

Scharlach, A., Graham, C. et Lehning, A. (2012). " The "village" model: A consumer driven approach for aging in place », The Gerontologist, vol. 52, n 3, p. 418-427.

Singelenberg, J., Stolarz, H. et McCall, M. (2014). «Integrated service areas: An innovative approach to housing, services and supports for older persons ageing in place », Journal of Community \& Applied Social Psychology, vol. 24, n 1, p. 69-73.

Statistique Canada. (2012). La situation des personnes âgées dans les ménages, 2012, Ottawa, Statistique Canada. [http://www12.statcan.gc.ca/census-recensement/2011/as-sa/98-312-x/98-312x2011003_4-fra.cfm]

Statistique Canada. (2017). Chiffres selon l'âge et le sexe, et selon le type de logement. Faits saillants du Recensement 2016, Ottawa, Statistique Canada. [http://www.statcan.gc.ca/dailyquotidien/170503/dq170503a-fra.htm?HPA=1]

Szanton, S., Thorpe, R., Boyd, C., Tanner, E. et Leff, B. (2011). « Community aging in place, advancing better lives for elders: A bio-behavioral-environmental intervention to improve function and health related quality of life in disabled older adults ", Journal of the American Geriatrics Society, vol. $59, \mathrm{n}^{\circ} 12$, p. 2314-2320.

Turcotte, M. (2014). Les Canadiens dont les besoins en soins à domicile sont non comblés, Ottawa, Statistique Canada. [http://www.statcan.gc.ca/pub/75-006-x/2014001/article/14042-fra.htm]

Vézina, M. et Crompton, S. (2012). Volunteering in Canada, Ottawa, Statistique Canada.

Wright, L., Vance, L., Sudduth, C. et Epps, J. (2015). « The impact of a home delivered meal program on nutritional risk, dietary intake, food security, loneliness, and social well-being », Journal of Nutrition in Gerontology and Geriatrics, vol. 34, n² 2, p. 218-227. 